\title{
Structural Characterization of Thin Epitaxial GaN Films on Polymer Polyimides Substrates by Ion Beam Assisted Deposition
}

\author{
Sri Vidawati ${ }^{1}$, Jürgen W. Gerlach ${ }^{2}$, Benjamin Herold², Bernd Rauschenbach ${ }^{2}$ \\ ${ }^{1}$ Faculty of Post Graduate, National Institute of Science and Technology, Jakarta 12640, Indonesia \\ ${ }^{2}$ Leibniz Institute of Surface Engineering (IOM), Permoserstrasse 15, D-04318 Leipzig, Germany \\ Email: juergen.gerlach@iom-leipzig.de
}

How to cite this paper: Vidawati, S., Gerlach, J.W., Herold, B. and Rauschenbach, B. (2020) Structural Characterization of Thin Epitaxial GaN Films on Polymer Polyimides Substrates by Ion Beam Assisted Deposition. Advances in Materials Physics and Chemistry, 10, 199-206.

https://doi.org/10.4236/ampc.2020.109015

Received: June 24, 2020

Accepted: September 24, 2020

Published: September 27, 2020

Copyright (c) 2020 by author(s) and Scientific Research Publishing Inc. This work is licensed under the Creative Commons Attribution International License (CC BY 4.0).

http://creativecommons.org/licenses/by/4.0/

\begin{abstract}
The Epitaxial GaN thin films have been fabricated by Ion Beam Assisted Deposition (IBAD) process using nitrogen ions with hyperthermal energies on the polyimides polymer substrates. By applying with the Reflection of High-Energy Electron Diffraction (RHEED), Scanning Electron Microscopy (SEM) and Quantum Design Physical Properties Measurement System, the behaviour of hexagonal GaN thin films is investigated. The result showed that the high quality of the deposited GaN layers kept appearing for many parameters depending on the temperature greatly. The behaviour of high quality of epitaxial GaN coating on the polyimide polymer substrates is a promising material for optoelectronic devices and semiconductor devices application.
\end{abstract}

\section{Keywords}

Ion Beam Assisted Deposition, Hexagonal GaN Thin Film, SEM, Quantum Design Physical Properties Measurement System, RHEED

\section{Introduction}

Thin film of $\mathrm{GaN}$ is a prospering material application for semiconductor devices [1] [2] and optoelectronic devices [3]. Gallium nitride is a binary III/V semiconductor occurring in three crystalline modifications: A hexagonal structure as the wurtzite polytype ( $\mathrm{w}-\mathrm{GaN})$, and two different cubic structures as the zinc blende polytype (z-GaN) and the $\mathrm{NaCl}$ structure (c-GaN) [4].

The most cases of thin film semiconductor are grown epitaxial on crystalline 
material, the growth models focus on regular lattice structures as substrates. By contrast, for the deposition on polymer materials, the semi-crystalline or amorphous character of these materials has to be considered. The interactions between moderately reactive metal atoms or non-metal ions and polymers are generally much weaker than the strong atomic or ionic bonds within the formed layers [5]. As a result, not only energy activation for adsorption and diffusion decreased, but also stored.

The growth of thin, high quality GaN layers on polyimide substrates is of great interest. The basis for the construction of semiconductor devices on polymers is the understanding of the growth mechanisms taking place especially in the initial stages of the film formation.

Here, the special variant of the IBAD technology, Ion Beam Assisted Molecular Beam Epitaxy (IBA-MBE) deployed to prepare the thin GaN films with high crystalline quality. It can be proved that this method is very successful for the direct growth of epitaxial GaN films [6] [7] [8]. In IBA-MBE, low energetic nitrogen ions $(<100 \mathrm{eV})$ are delivered to the surface of the growing thin film during the deposition process.

In this paper, we report the growth of thin gallium nitride $(\mathrm{GaN})$ on the polyimide polymer substrates by Ion Beam Assisted Deposition process (IBAD) using nitrogen ions with hyperthermal energies. The Reflection High-Energy Electron Diffraction (RHEED), Scanning Electron Microscopy (SEM), and Quantum Design Physical Properties Measurement System were carried out to investigate the behaviour of the thin film $\mathrm{GaN}$ on the polymer substrates. As this work is focused on the growth of technical relevant hexagonal GaN, the denotation gallium nitride means the wurtzite polytype in the following.

\section{Materials and Methods}

The thin films of GaN were grown on Kapton type polyimide substrates by ion beam assisted deposition (IBAD) process interconnected ultra-high vacuum chambers for the film deposition including reflection of high-energy electron diffraction (RHEED) for in situ studies of film growth. The ion beam assisted deposition of $\mathrm{GaN}$ on polyimide equals in principle the Ion Beam Assisted Molecular Beam Epitaxy (IBA-MBE) process described in former works [6]. The only difference is the amorphous character of the polyimides, which leads to a non-epitaxial growth. The residual gas pressure prior deposition was $10^{-8} \mathrm{~Pa}$. Ga was deposited by an effusion cell at temperatures between $950^{\circ} \mathrm{C}$ and $1050^{\circ} \mathrm{C}$. In this temperature range the deposition rate $\Phi \mathrm{Ga}$ varied between $5 \times 10^{13}$ and $2 \times 10^{14} \mathrm{Ga}$ atoms $/ \mathrm{cm}^{2}$ according to the calibration relation $\Phi \mathrm{Ga}=8 \times 10^{7} \times \exp (\mathrm{TEC} / 71)$ obtained by Rutherford backscattering spectroscopy (TEC is the temperature of the effusion cell). The energy of these hyperthermal ions between $20 \mathrm{eV}$ until 50 $\mathrm{eV}$, and the nitrogen ion flux of $1.6 \times 10^{14} \mathrm{ions} / \mathrm{cm}^{2}$ was kept constant.

As a substrates material is a type of Kapton polyimide. Polyimides in general are polymers consisting of at least two imide monomers that are arranged in a 
molecular chain. Chemically the imide monomer is built-up of two nitrogen bound acyl groups. The chemical structure is shown in Figure 1.

Polyimides exhibit very high thermo stability compared to other polymers. Consequently, for GaN deposition, where the high substrate temperatures should be close to the required $700^{\circ} \mathrm{C}$, the most stable thermal polyimide aromatic was chosen. Poly $\left(4,4^{\prime}\right.$-oxydiphenylene-pyromellitimide), produced by the company DuPont as Kapton, has a glass transition temperature of about $360^{\circ} \mathrm{C}$, is a short time heat-resistant up to $400^{\circ} \mathrm{C}$.

The thin film deposition process was monitored in situ by reflection high energy electron diffraction (RHEED). During deposition of the layer, the working pressure reaches merely $8 \times 10^{-2} \mathrm{~Pa}$. For that purpose an electron beam with a selectable energy between $10 \mathrm{keV}$ and $30 \mathrm{keV}$ grazes the sample under a small angle $\left(<2^{\circ}\right)$ to the surface. Electrons are reflected or diffracted on the surface and hit the fluorescent RHEED screen.

The surface topography of the grown thin film of GaN was investigated ex situ with Scanning Electron Microscopy (SEM). The images were collected by the SE analysis with an FEI Quanta 250 microscope with Everhart-Thornley detector (EDT) and a Zeiss AURIGA with a lens detector, while the electron acceleration voltages between $2 \mathrm{kV}$ and $10 \mathrm{kV}$ was applied.

The resistivity of the thin film of $\mathrm{GaN}$ on polyimides substrate was investigated ex situ with the Quantum Design Physical Properties Measurement System EverCool-II ${ }^{\mathrm{TM}}$ at room temperature. Resistivity measurement used a four-probe lead configuration method. Thin film of $\mathrm{GaN}$ on polyimides prepared to be mounted in a four-probe configuration that used gold wire in each probe (the thickness of the gold wire is just like the thickness of human hair). We used the glue of silver loaded epoxy hardener 186-3616 and silver loaded epoxy adhesive 186 - 3616 (glue used for gold wire on the surface of thin film GaN). The glues mixed then paste on the surface of the thin film GaN in four-probe configurations. The gold wire was placed for each probe. We have to wait for $12-24$ hours until the glue was drained. Then the gold wire should be useful solder for contact between the thin film of GaN and the sample holders.

\section{Results and Discussion}

We were studying the growth of high-quality the thin film of $\mathrm{GaN}$ on polyimide substrates. The basis for the construction of semiconductor devices on the<smiles>[R]C(=O)N([R])C([R])=O</smiles>

(a)

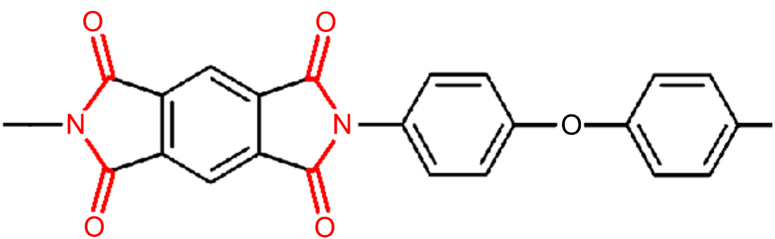

(b)

Figure 1. Chemical structure: (a) an imide monomer with Rn open bond, and (b) the chemical structure of Polyimides. 
polymers understanding of the growth mechanisms that occur especially in the initial stages of film formation. In this work, thin film of $\mathrm{GaN}$ were grown on Kapton type polyimide substrates by ion beam assisted deposition (IBAD) process using nitrogen ions with hyperthermal energies. The deposited thin film of $\mathrm{GaN}$ as well as the polymer substrates were analyzed by various methods in situ and ex situ. This experiment was initially describe the method of analysis, beginning with the in situ RHEED measurements, followed ex situ by surface topography characterization with SEM, and resistivity measurement with Quantum Design Physical Properties Measurement System. Afterwards, different samples were prepared with varying growth parameters, especially various at the substrate temperature during deposition and deposition time. The resulting substrate temperature and film thickness series were investigated.

The resulting RHEED pattern is mostly originating from the elastically scattered electrons. Compared to them, emitted secondary electrons and inelastic scattered electrons have largely very low energy, so no additional energy filter is required. For GaN deposition on amorphous substrates such as polyimide no epitaxial growth is expected, but the film consists of different crystallites with varying orientations. In this case the rods in the reciprocal space, according to individual crystallites, can be viewed as a set of concentric spheres. The intersection of Ewald sphere with each reciprocal sphere resulted a circle, called the Debye ring. As a result for the polycrystalline materials the arches in the RHEED pattern are completely "filled" and the ring pattern with the incident beam in the centre can be observed [9] (see Figure 2).

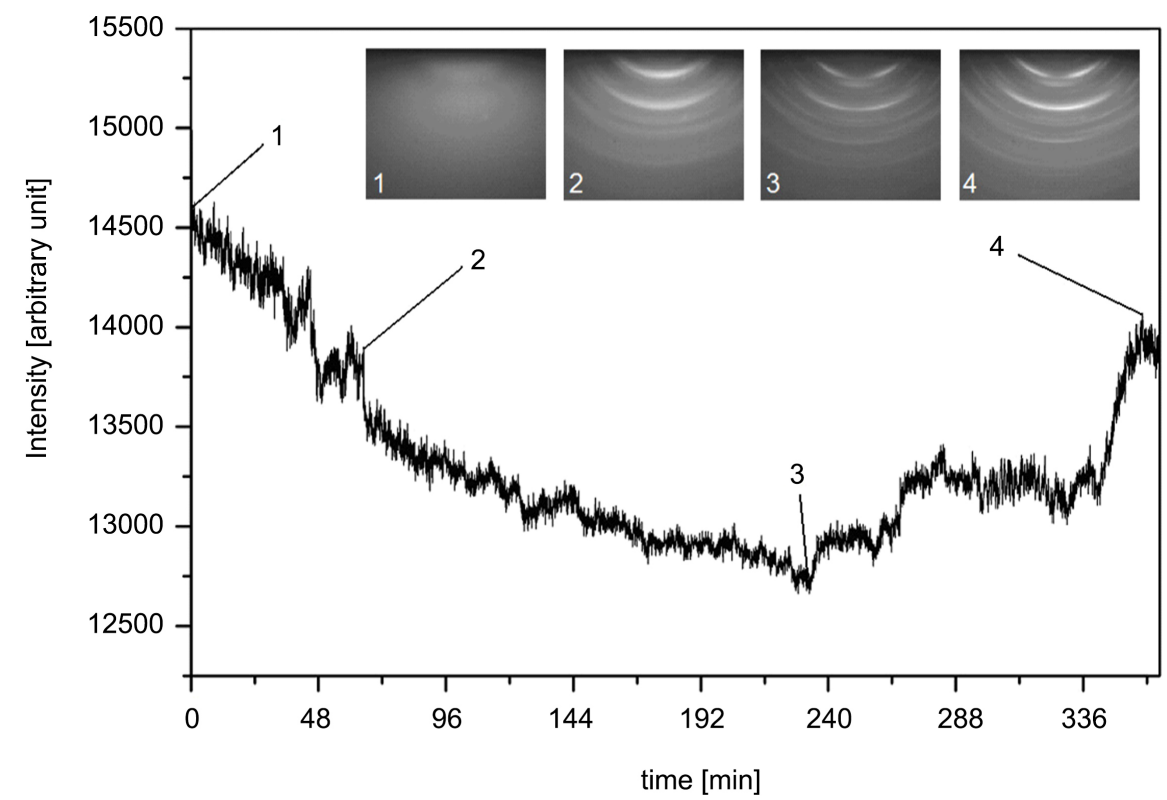

Figure 2. RHEED pattern intensity development during deposition and postnitridation of thin film of GaN on Kapton type polyimide substrate: 1) start of the deposition; RHEED pattern of the Kapton surface, 2) proceeding deposition; emerging RHEED pattern of a polycrystalline structure, 3) end of the deposition, start of the postnitridation; intensity minimum, 4) end of the postnitridation, intensity maximum. 
The surface topography of the grown thin film of GaN was investigated ex situ with SEM to obtain a qualitative impression of the layer quality and surface roughness, see Figure 3 and Figure 4. The image up to a magnification of $120,000 \mathrm{x}$ is possible. Above this magnification fill effect affected electrons and cause the drift effect in imaging system and blurred effects. At higher acceleration voltages the Cirlex substrate was significantly damaged by the electrons.

The characterization resistivity of $\mathrm{GaN}$ on polymer polyimides with various parameters of deposition had been measured. The result of resistivity of GaN on polymer polyimide was shown in Figure 3 and Figure 4. The results of thin film of $\mathrm{GaN}$ on polymer polyimide substrates were analyzed and discussed to compared with the former experiments with the measurement of resistivity by Quantum Design Physical Properties Measurement System and topography characterization (grain boundary) of the results by SEM.
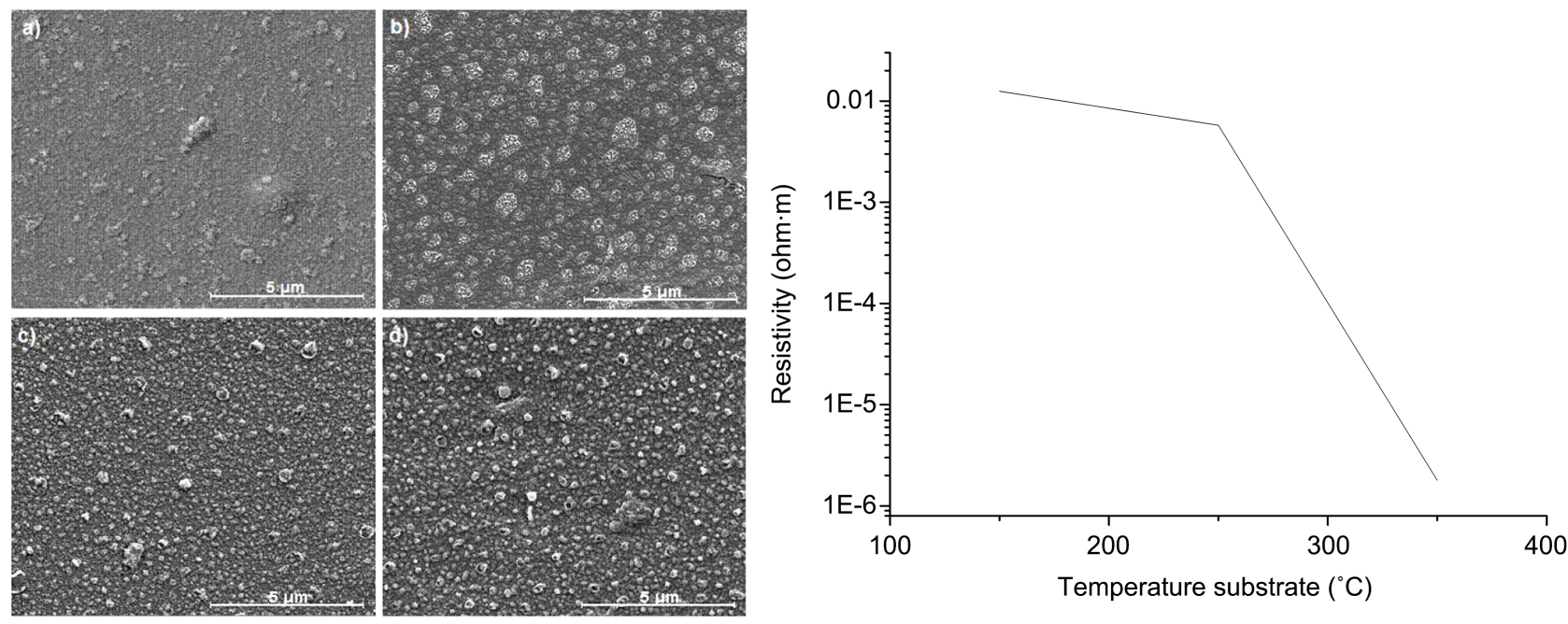

Figure 3. Surface topography and resistivity of $\mathrm{GaN}$ layers deposited on the polyimides with various temperatures of substrates (a) $350^{\circ} \mathrm{C}(\mathrm{b}) 250^{\circ} \mathrm{C}$ (c) $150^{\circ} \mathrm{C}$ and (d) without heating $\left(\approx 67^{\circ} \mathrm{C}\right)$.
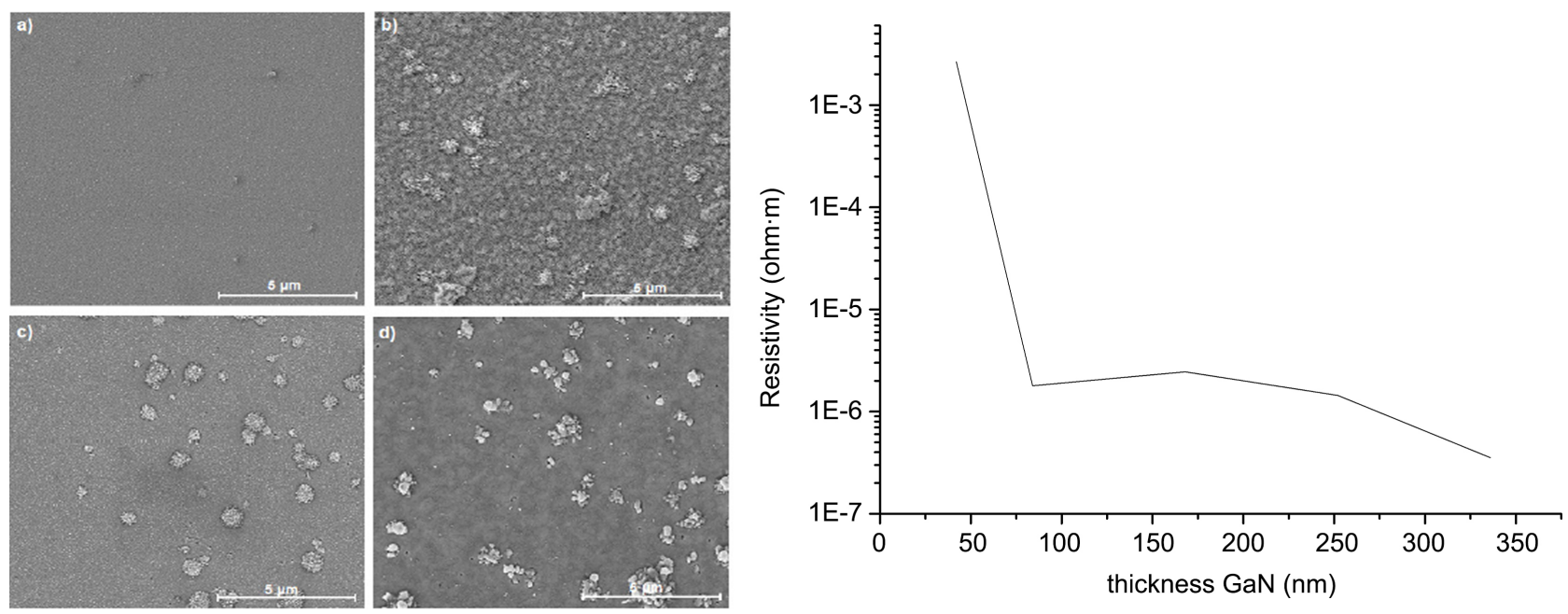

Figure 4. Surface topography and resistivity of GaN layers on the polyimides with various thickness of GaN layers: (a) $42 \mathrm{~nm}$, (b) $84 \mathrm{~nm},(\mathrm{c}) 168 \mathrm{~nm}$ and (d) $336 \mathrm{~nm}$. 
From resistivity measurement used Quantum Design Physical Properties Measurement System EverCool- $\mathrm{II}^{\mathrm{TM}}$ at the room temperature (see in Figure 3). We found the resistivity of $\mathrm{GaN}$ on polymer polyimides decrease very sharp when the substrate temperature changed from $250^{\circ} \mathrm{C}$ to $350^{\circ} \mathrm{C}$. We compared between resistivity characterized results with topography characterized result. SEM characterized showed (see in Figure 3) an overview over the surfaces of the temperature series samples.

For $350^{\circ} \mathrm{C}$ substrate temperature (see in Figure 3(a)) the general topography of the surface emerges as rather smooth, showing a homogenous distribution of small crystallites with detached larger crystallite aggregates on top. The comparable high temperature implies increased diffusion leading to a smooth build-in of surplus large gallium droplets during post nitridation, while the irregular shape and distribution of the on-top aggregates hints on overgrown particles which served as seeds for crystal growth. For a reduced substrate temperature of $250^{\circ} \mathrm{C}$ (see in Figure $3(\mathrm{~b})$ ) the surface consists of stacked islands of different sizes, displaced by each other. These islands are remains of gallium droplets on the top of the layer, which was converted to GaN during post nitridation. Despite the substrate temperature decrease and thereby lower of the surface diffusion, the smaller gallium droplets can be assemble into larger ones. For lower substrate temperatures of $150^{\circ} \mathrm{C}$ (see in Figure $3(\mathrm{c})$ ) down to $67^{\circ} \mathrm{C}$ (see in Figure $3(\mathrm{~d})$ ), a very rough surface can be observed.

Figure 4 shows the surfaces of $42 \mathrm{~nm}, 84 \mathrm{~nm}, 168 \mathrm{~nm}$ and $336 \mathrm{~nm}$ thick films. Since all the layers were deposited at $350^{\circ} \mathrm{C}$ substrate temperature, their surfaces are comparably smooth, showing the homogenous distribution of small crystallites and separate aggregates describe before. Compared with polycrystalline layers on PI, the crystalline quality and surface topography of epitaxial GaN layers grown on single crystalline substrates strongly depends on their thickness. Here, especially line- and area defects are overgrown with increasing thickness [10]. In contrast, the layers grown on PI consist of numerous small crystallites from the initial stages on; accordingly, the layer quality does not change significantly with increase of the layer thickness.

The resistivity analysis by Quantum Design Physical Properties Measurement System EverCool-II ${ }^{\mathrm{TM}}$ at the room temperature, we shown that resistivity of GaN on polymer polyimide decreased drastically when the thickness of thin film GaN on polymer polyimide increase from $42 \mathrm{~nm}$ to $84 \mathrm{~nm}$, and then decreased resistivity relative linear when the thickness of thin film $\mathrm{GaN}$ was increase.

The result shown a temperature-dependent chemical composition of the layers: The concentrations of gallium and nitrogen are reduced in favour of oxygen as a result of the roughened surface and voids, which offer more surface area for oxidation. From the following SEM investigations the direct influence of the substrate temperature during deposition on the roughness and porosity of the layers is derived. The analysis of the surface topography proved the direct influence of the substrate temperature during deposition in roughness and porosity 
of the layers, which increased from the layers deposited at maximum temperature of $350^{\circ} \mathrm{C}$ down to layers deposited near room temperature. As a reason for this, decreased surface diffusion and desorption of gallium were named, leading to the growth of smaller crystallites and to surplus gallium atoms on the surface. No significant layers that depend on the surface topography change were found for the thickness range of layers is investigated.

\section{Conclusion}

The growth of thin, high quality GaN layers on polyimide substrates is of great interest. The basis for the construction of semiconductor devices on polymers is the understanding of the growth mechanisms taking place especially in the initial stages of the film formation. Wurtzite nanocrystallite GaN thin film was successfully grown on the polymer polyimide substrates via IBAD process using nitrogen ions with hyperthermal energies. The result demonstrated that the quality of the deposited GaN layers emerged for many parameters strongly temperature dependent. The optimum conditions for epitaxial growth and high quality of $\mathrm{GaN}$ layers on the polyimide substrates useful for optoelectronic devices and semiconductor devices applications. Furthermore, some practical purposes and application still need to be done in the future studies.

\section{Acknowledgements}

This study was supported by the Deutscher Akademischer Austauschdienst (DAAD, SV).

\section{Conflicts of Interest}

The authors declare no conflicts of interest regarding the publication of this paper.

\section{References}

[1] Manasreh, O. (2000) III-Nitride Semiconductors: Electrical, Structural and Defect Properties. Elsevier: Amsterdam, The Netherlands.

[2] Nomura, K., Ohta, H., Takagi, A., Kamiya, T., Hirano, M. and Hosono, H. (2004) Room-Temperature Fabrication of Transparent Flexible Thin-Film Transistors Using Amorphous Oxide Semiconductors. Nature, 432, 488-492. https://doi.org/10.1038/nature03090

[3] Ellmer, K. (2000) Magnetron Sputtering of Transparent Conductive Zinc Oxide: Relation between the Sputtering Parameters and the Electronic Properties. Journal of Physics D: Applied Physics, 33, R17-R32. https://doi.org/10.1088/0022-3727/33/4/201

[4] Wang, L.D. and Kwok, H.S. (2000) Cubic Aluminum Nitride and Gallium Nitride Thin Films Prepared by Pulsed Laser Deposition. Applied Surface Science, 154-155, 439-443. https://doi.org/10.1016/S0169-4332(99)00372-4

[5] Faupel, F., Zaporojtchenko, V., Thran, A., Strunskus, T. and Keine, M. (2004) Metal Diffusion in Polymers and on Polymer Surfaces. In: Diffusion Processes in Advanced Technological Materials, Norwich, William Andrew Inc., 550. 
https://doi.org/10.1016/B978-081551501-2.50009-5

[6] Finzel, A., Gerlach, J.W., Lorbeer, J., Frost, F. and Rauschenbach, B. (2014) High-Fluence Hyperthermal Ion Irradiation of Gallium Nitride Surfaces at Elevated Temperatures. Applied Surface Science, 317, 811-817.

https://doi.org/10.1016/j.apsusc.2014.09.006

[7] Greene, J.E., Barnett, S.A., Sundgren, J. and Rockett, A. (1989) Low-Energy Ion/Surface Interactions during Film Growth from the Vapor Phase. In: Itoh, T., Ed., Ion Beam Assisted Deposition, Elsevier, Amsterdam, The Netherlands, 105-152. https://doi.org/10.1016/B978-0-444-87280-7.50010-9

[8] Rauschenbach, B., Lotnyk, A., Neumann, L., Poppitz, D. and Gerlach, J.W. (2017) Ion Beam Assisted Deposition of Thin Epitaxial GaN Films. Materials, 10, 690. https://doi.org/10.3390/ma10070690

[9] Andrieu, S. and Fréchard, P. (1996) What Information Can Be Obtained by RHEED Applied on Polycrystalline Films. Surface Science, 360, 289-296. https://doi.org/10.1016/0039-6028(96)00303-2

[10] Gerlach, J.W., Sienz, S., Attenberger, W. and Rauschenbach, B. (2001) Influence of Defects in Low-Energy Nitrogen Ion Beam Assisted Gallium Nitride Thin Film Deposition. Physica B, 308-310, 81-84.

https://doi.org/10.1016/S0921-4526(01)00661-5 